\title{
Síndrome Nefrótico idiopático en niños tratados con ciclofosfamida
}

\author{
Heerlein A., ${ }^{*}$ Corsini N., * Rodriguez E., ,* Puga F.**
}

\begin{abstract}
A total of 31 children with nephrotic syndrome were treated with tyclophosfamide $2-3 \mathrm{mg} / \mathrm{kg}$. day for two months.

Good results were observed in $75 \%$ of the patients with frequent relapses and in $60 \%$ of the patients resistent to the steroid therapy.

Only in 6 patients (19.5\%) the toxic effects of the drug were observed.
\end{abstract}

El Síndrome Nefrótico es consecuencia de múltiples etiologías y sustratos histológicos diferentes, siendo la respuesta al tratamiento clásico con corticoides, en cierto grupo de pacientes, ineficaz. ${ }^{1}$ Algunos de ellos son inicialmente sensibles a la terapia esteroidal, pero presentan recaídas frecuentes. Otros son inicialmente resistentes a los corticoides pudiendo establecerse una correlación (no lineal) entre la respuesta a la terapia esteroidal, la forma sintomática de presentación (Sindrome Nefrótico puro) y el sustrato histológico.

El uso de ciclofosfamida en pacientes dependientes o resistentes a la terapia esteroidal fue intraducido por Moncrieff, obteniendo una respuesta muy favorable en el primer grupo mencionado. ${ }^{3}$

Otros autores reportan resultados similares señalando, además, la presencia de efectos tóxicos del tratamiento con ciclofosfamida en dosis entre 4 y $6 \mathrm{mg} / \mathrm{kg}$ de peso.2. 4.8 La toxicidad de la droga puede manifestarse de múltiples formas incluyendo la cistitis hemorrágica, alopecia, leucopenia y otras. ${ }^{7}$ Se han descrito casos de esterilidad en nin̄os tratados en etapa prepuberal, posiblemente causados por ciclofosfamida.

El presente trabajo intenta determinar la eficacia del uso de ciclofosfamida en dosis menores (2$3 \mathrm{mg} / \mathrm{kg}$ ) en el tratamiento del Sindrome Nefrótico con el objeto de obtener una reducción significativa en la aparición de efectos tóxicos inherentes a la droga.

*Internos de 7. Año, Facultad de Medicina Sede Oriente *"Departamento de Nefrourologis. Hospital Luis Calvo Mackenna.

\section{MATERIAL Y METODO}

De un total de 148 pacientes portadores de Sindrome Nefrótico Idiopático atendidos entre 1974-1979, se analiza una muestra de 31 nin̄os (20.9\%), 16 hombres y 15 mujeres, tratados con ciclofosfamida a razón de $2-3 \mathrm{mg} . / \mathrm{kg}$. durante dos meses. En 20 casos se asoció prednisona en dosis de $\mathbf{l m g} . / \mathrm{kg}$. La distribución segin la edad fluctuó entre los 9 meses y los 12 años con una edad promedio de 4.l años (Tabla l). La suspensión de las drogas se efectuó bruscamente al completar el período señalado, controlándose a los pacientes por un lapso promedio de 17.4 meses. El diagnóstico de S. Nefrótico se estableció de acuerdo al cuadro clínico y a los exámenes complementarios. Se efectuó por lo menos un estudio histologico en cada caso, obtenido por punción-biopsia renal percutánea. Del total, 8 pacientes eran dependientes a los esteroides (sensibles con 3 o más recaidas en 12 meses) y resistentes los 23 restantes. La Tabla 2 los correlaciona con el tipo histológico, predominando la Lesión Mínima (22 casos) seguida por hialinosis focal (5 casos) y glonerulonefritis crónica (2 casos).

Dentro de los 8 casos clasificados como dependientes 7 presentaban un sustrato histológico compatible con Lesión Mínima (Tabla 3).

En cuanto a la forma sintomática de presentación $20 \%$ de los pacientes presentaron hipertensión arterial y $38 \%$ hematuria micro y 10 macroscópica. Ambas manifestaciones se registraron en un pequeño número de pacientes con lesión mínima, en los que la hematuria siempre fue microscópica y la hipertensión, transitoria. Por el contrario, en aquellos con GNC y hialinosis dichas manifestaciones estuvieron presentes en casi todos los casos y por lapso prolongado. 


\section{RESULTADOS Y COMENTARIO}

Se considexó buen resultado del tratamiento aquel enfermo que no recayó en los períodos de control señalados anteriormente.

Los resultados fueron buenos en el $75.0 \%$ de los pacientes dependientes $y$ en un $60 \%$ de los resistentes a la terapia esteroidal (Tabla 4), con una respuesta favorable global de $67 \%$, Los resultados fueron significativamente favorables en los pacientes con LM y malos en los portadores de GNC. (Tabla 5).

En cuanto a la asociación de ciclofosfamida y prednis ona no encontramos diferencias significativas en la respuesta respecto a los 11 casos tratados sólo con ciclofosfamida.

Del total de pacientes sólo 5 presentaron leucopenia $(16 \%)$ y 1 , cistitis hemorrágica, sin evidenciarse otras complicaciones. Creemos por lotanto que el uso de ciclofosfamida en las dosis mencionadas constituye una buena posibilidad tera-

Tabla N." 1

Distribución por Grupos Etarios

$\begin{array}{lcccccc} & \text { días } & \text { meses } & \text { meses } & \text { años } & \text { años } & \text { años } \\ \text { Edad } & 0-28 & 1-11 & 12-24 & 2-5 & >5 & >10 \\ \text { N. }{ }^{0} \text { casos } & 0 & 2 & 8 & 12 & 7 & 2 \\ \% & & 6.4 & 25.8 & 38.7 & 22.5 & 6.4\end{array}$

péutica en los pacientes con S.N.I. dependientes o resistentes a los esteroides, ya que se obtienen resultados similares a los obtenidos con dosis mayojes pero, con una clara disminución en la ìncidencia de complicaciones.

Finalmente queremos destacar la necesidad de contar con el diagnóstico histológico antes de iniciar la terapia con ciclofosfamida, ya que la respuesta a éste depende principalmente del tipode lesión histológica encontrada.

\section{RESUMEN}

Se analizan 31 pacientes con Síndrome Nefrótico sensibles con recaidas frecuentes y resistenles a la terapia esteroidal tratados con ciclofosfamida $2-3 \mathrm{mg} . \mathrm{kg}$. por 2 meses. Se observa una respuesta favorable tanto en los enfermos sensibles con recaidas frecuentes, como en los resistentes junto con una menor frecuencia de aparición de efectos tóxicos inherentes al uso de ciclofosfamida.

Tabla N. ${ }^{\circ}$

Resultados del Tratamiento con Ciclofosfamida versus respuesta a la Terapia Esteroidal

$\begin{array}{lccccr}\text { Resultado } & \text { Bueno } & \% & \text { Malo } & \% & \text { Total } \\ \text { Dependiente } & 6 & 75.0 & 2 & 25.0 & 8 \\ \text { Resistente } & 14 & 60.9 & 9 & 39.1 & 23 \\ \text { Total } & 20 & 64.5 & 11 & 35.5 & 31\end{array}$

Tabla N. 2

Clasificación Histologica

\begin{tabular}{|c|c|c|c|c|c|}
\hline $\begin{array}{c}\text { Tipo de } \\
\text { Lesión } \\
\text { N. }{ }^{\circ} \text { casos } \\
\%\end{array}$ & $\begin{array}{c}\text { Lesión } \\
\text { Minima } \\
22 \\
70.96\end{array}$ & $\begin{array}{c}\text { Exudativa } \\
1 \\
3.22\end{array}$ & $\begin{array}{c}\text { Focal } \\
5 \\
16.15\end{array}$ & $\begin{array}{c}\text { Mesangial } \\
1 \\
3.22\end{array}$ & $\begin{array}{c}\text { Gomerulo- } \\
\text { Nefritis Cr } \\
2 \\
6.45\end{array}$ \\
\hline
\end{tabular}

Tabla N. ${ }^{\circ} 3$

Respuesta a la Terapia Esteroidal, según tipo Histológico

\begin{tabular}{|c|c|c|c|c|c|c|}
\hline $\begin{array}{l}\text { Tipo de } \\
\text { Lesión } \\
\text { Dependientes }\end{array}$ & $\begin{array}{c}\text { Lesión } \\
\text { Mínima } \\
7\end{array}$ & Exudativa & Focal & Mesangial & $\begin{array}{l}\text { Glomendo- } \\
\text { nefritis } \mathbf{C r}\end{array}$ & $\begin{array}{c}\text { Total } \\
8\end{array}$ \\
\hline Resistentes & 15 & $\mathbf{I}$ & 5 & & 2 & 23 \\
\hline
\end{tabular}

Tabla N. 5

Resultados del tratamiento con Ciclofosfamida versus tipo hitológico

\begin{tabular}{lcccccc} 
Tipo de & Lesión & & & & \multicolumn{3}{c}{ Glomerulo- } \\
Lesión & Mínima & Exudativa & Focal & Mesangial & nefritis Cr & Total \\
R Bueno & 14 & 1 & 3 & 1 & 1 & 20 \\
R Malo & 8 & 1 & 2 & 1 & 1 & $\mathbf{1 1}$ \\
Total & 22 & & 5 & 1 & 2 & 31
\end{tabular}




\section{REFERENCLAS}

ISymposiun of the Vietrotic Syndrone J. of Pediatrics 58: 607 May 1961.

2"Experience whith alkylating agents in the treament of childien whith Nephrotic Syndrome". Med. Jou. 59/24 (987-969) 1963.

3Morcrie $f f$ N.W. et ah "Cyclofoslamide therapy in the Nephrotic Syndrome”. Brit. Med. Jou. 666, 1969.

tWe st C.D. "Effect of cyclofoslanide in lipoid nephrosis in the human and on aminoglucoside nephrosis in the rat". Jou of Pediat. 608: 516, april 1968.
${ }^{5}$ Ehel ford $C . N$. "Cychophosfarnide in the treatment of Idiopathic Lipoid Nephrosis". Jou of Pediat. 70: 758 May 1968.

6Sindrome Nefrótico Idiopático en el niño. Puga F., Hodriguez E. Rev. Chilena de Pedintria Vol. 2 Marzo 1974.

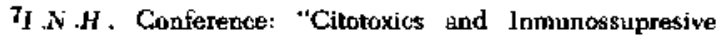
dnugs in non tomor process". Am. Jou. of Internal Medicine 165: 183, 1972 .

8Inmunosupresores en el sindrome nefrotico. Puga F., Rodrdguez E., Rosenberg H. Rev. Chilena de Pediatria Vol. 45, pág. $221-223,1974$. 